\title{
Epigallocatechin gallate promotes p53 accumulation and activity via the inhibition of MDM2-mediated p53 ubiquitination in human lung cancer cells
}

\author{
LONGYU JIN $^{1}$, CUI LI ${ }^{2}$, YAN XU ${ }^{2}$, LI WANG ${ }^{3,4}$, JIANXIN LIU ${ }^{1}$, DIANJUN WANG ${ }^{1}$, \\ $\mathrm{CAO}_{\text {HONG }}{ }^{1}, \mathrm{ZHIBING} \mathrm{JIANG}^{1}$, YUCHAO MA ${ }^{4}$, QIAN $\mathrm{CHEN}^{4}$ and FENGLEI YU ${ }^{4}$ \\ ${ }^{1}$ Cardiothoracic Surgery, The Third XiangYa Hospital, Central South University, Changsha, \\ Hunan 410013; ${ }^{2}$ Key Laboratory of Cancer Proteomics of Chinese Ministry of Health, Xiangya Hospital, \\ Central South University, Changsha, Hunan $410008 ;{ }^{3}$ Cancer Research Institute, Xiang Ya Medical School, \\ Central South University, Changsha, Hunan $410078 ;{ }^{4}$ Cardiothoracic Surgery, The Second \\ Xiang Ya Hospital, Central South University, Changsha, Hunan 410011, P.R. China
}

Received November 19, 2012; Accepted December 30, 2012

DOI: $10.3892 /$ or.2013.2343

\begin{abstract}
Epigallocatechin gallate (EGCG), which is derived from green tea, is well known for its chemopreventive activity. Several studies have shown that p53 plays an important role in the activity of EGCG; however, the mechanism by which EGCG regulates p53 requires further investigation. In the present study, we showed that EGCG inhibits anchorage-independent growth of human lung cancer cells by upregulating p53 expression. EGCG treatment can substantially increase p53 stability, promote nuclear localization of p53 and decrease nuclear accumulation of MDM2. We also found that EGCG increases the phosphorylation of p53 at Ser15 and Ser20 and enhances its transcriptional activity. Although EGCG promotes MDM2 expression in a p53-dependent manner, the interaction between MDM2 and p53 was significantly inhibited following EGCG treatment, which resulted in the inhibition of MDM2mediated p53 ubiquitination. Thus, our results suggest that the stabilization and activation of p53 may partly contribute to the anticancer activity of EGCG.
\end{abstract}

\section{Introduction}

According to the results of epidemiologic studies, green tea consumption has a preventive effect on carcinogenesis (1-6). It is thought that polyphenols, also known as catechins, play an important role in the chemopreventive effects mediated by

Correspondence to: Professor Fenglei Yu, Cardiothoracic Surgery, The Second XiangYa Hospital, Central South University, 139 Renmin Road, Furong, Changsha, Hunan 410011, P.R. China E-mail: feng12nd@gmail.com

Abbreviations: EGCG, epigallocatechin gallate, $\mathrm{CHX}$, cycloheximide;

Key words: epigallocatechin gallate, anchorage-independent growth, p53, MDM2 green tea. (-)-Epigallocatechin gallate (EGCG), a type of polyphenol, which is the most well known, abundant and active compound found in green tea, exerts its anticancer effects in a wide range of malignancies $(2,7)$. Previous studies have suggested that multiple signaling pathways and mechanisms are involved in the antitumor activity of EGCG (1,8-10), including suppression of various protein kinases (11-13); disruption of the activation of transcription factors such as EGFR, NF- $\kappa$ B, AP-1 and STATs (14-16); induction of cell cycle arrest or apoptosis $(17,18)$; and inhibition of cell migration and metastasis (19-24).

p53, commonly referred to as the 'cellular gatekeeper' or 'the guardian of the genome', is a crucial tumor suppressor gene that is mutated in more than half of all types of human cancer. As a transcription factor, p53 functions to regulate cell fate following various types and levels of cellular stress through its downstream target genes. In addition to its canonical functions of inducing DNA repair, cell cycle arrest and apoptosis $(25,26)$, recent studies have also revealed that p53 is involved in the regulation of various other cellular functions, such as senescence, metabolism and autophagy. Due to the importance of $\mathrm{p} 53$, its activation is regulated by complicated post-translational modifications, such as phosphorylation, acetylation, ubiquitination and sumoylation (8,27-29). Previous studies have shown that the phosphorylation and acetylation of p53 promotes the expression of p53 target genes $(28,30,31)$, whereas other modifications, such as ubiquitination and sumoylation, are considered to be associated with the suppression of p53-mediated transcription and nuclear export of p53 (32-34). MDM2, a Ring finger domain-containing protein, is the first identified E3 ligase that can induce p53 ubiquitination and proteasomal degradation (33). However, as a transcriptional target of p53, MDM2 expression is also regulated in a p53-dependent manner. As p53 increases, the expression of MDM2 is strengthened, which induces degradation of p53 and achieves balance.

Previous studies have demonstrated that EGCG treatment increases the expression levels of p53 in various human cancer 
cells (35-38). Previous reports found that cells expressing wildtype p53 are more sensitive than p53-null or p53 knock down cells to EGCG-induced growth inhibition and apoptosis $(36,39,40)$. However, the molecular mechanisms underlying p53 regulation by EGCG and the anticancer effects of green tea via targeting of the p53 tumor suppressor gene are poorly understood. In the present study, we demonstrated that EGCG treatment can induce p53 accumulation and enhance the stability of this protein by disturbing the interaction between p53 and MDM2. Furthermore, our data showed that EGCG inhibits p53 ubiquitination in a dose-dependent manner. These results indicate a novel mechanism for the preventive effects of EGCG on cancer.

\section{Materials and methods}

Cell culture and transfection. All cell lines were obtained from the American Type Culture Collection and were grown in a $37^{\circ} \mathrm{C}$ incubator with $5 \% \mathrm{CO}_{2}$ according to the American Type Culture Collection protocols. For transfection experiments, the Lipofectamine ${ }^{\mathrm{TM}} 2000$ transfection reagent (Invitrogen, Carlsbad, CA, USA) was used according to the manufacturer's instructions.

Reagents and antibodies. EGCG, cycloheximide (CHX) and MG132 were obtained from Sigma (St. Louis, MO, USA). The GFP-p53, His-Ub and HA-MDM2 plasmids were gifts from Dr Bo Liu and were previously described (41). Anti-p53, antiMDM2, anti- $\beta$-actin, anti-GFP, anti-HA, anti-His, anti-rabbit IgG-HRP, anti-mouse IgG-HRP, anti-goat IgG-HRP, and normal mouse/rabbit IgG were purchased from Santa Cruz Biotechnology (Santa Cruz, CA, USA). Anti-phospho-p53 (Ser15), anti-phospho-p53 (Ser20), anti-p21, anti-lamin B, antitubulin and anti-ubiquitin antibodies were purchased from Cell Signaling Technology, Inc. (Danvers, MA, USA).

Immunoprecipitation. $293 \mathrm{~T}$ and $\mathrm{H} 1299$ cells were first co-transfected with $2 \mu \mathrm{g}$ of GFP-p53 and $2 \mu \mathrm{g}$ of HA-MDM2. Twenty-four hours after the transfection, these cells were treated with $40 \mu \mathrm{M}$ EGCG for $24 \mathrm{~h}$. Cells were harvested and washed twice with ice-cold PBS and lysed in NP40 lysis buffer (50 mmol/1 Tris-HCl, pH 8.0; $150 \mathrm{mmol} / 1 \mathrm{NaCl} ; 0.5 \% \mathrm{NP} 40$ ) with protease cocktail (Roche Diagnostics GmbH, Mannheim, Germany). All immunoprecipitation procedures were carried out at $4^{\circ} \mathrm{C}$. The lysates were then incubated with the appropriate antibodies, followed by incubation with protein A/G agarose beads. The protein-antibody complexes were recovered and subjected to western blot analysis after separation by SDS-PAGE.

Western blotting. Cells were harvested by trypsinization and pelleted by centrifugation. Cell pellets were lysed in NP40 lysis buffer supplemented with protease inhibitors. Protein concentrations were determined using the Bradford assay (Bio-Rad Laboratories Philadelphia, PA, USA). Proteins were separated by SDS-PAGE and electrically transferred to a polyvinylidene difluoride membrane (Millipore, Billerica, MA, USA). After blocking in 5\% non-fat dry milk in TBS, the membranes were hybridized to specific primary antibodies overnight at $4^{\circ} \mathrm{C}$, washed three times with TBS Tween-20, and then incubated with secondary antibodies conjugated with horseradish peroxidase for $1 \mathrm{~h}$ at room temperature. Next, the membranes were washed three times in TBS Tween-20 at room temperature. The protein bands were visualized using ECL chemiluminescence reagents (Pierce Chemical Co., Rockford, IL, USA) according to the manufacturer's protocol.

Measurement of 53 and MDM2 half-lives. For p53 and MDM2 protein stability experiments, after the treatment of A549 cells with $40 \mu \mathrm{mol}$ of EGCG for $24 \mathrm{~h}, 30 \mu \mathrm{g} / \mathrm{ml}$ of CHX was added to inhibit protein synthesis. Subsequent time points for incubation in medium containing EGCG and CHX were 0, 20, 40, 60 or $90 \mathrm{~min}$ as indicated. Cells were then processed as previously described for western blotting and quantified by densitometry.

Luciferase reporter assay. The pGL3-p53 firefly luciferase reporter plasmid and the internal control pRL-SV40 (Renilla luciferase) plasmid were purchased from Promega. A549 cells were cultured in 24-well plates and co-transfected with the indicated plasmids using Lipofectamine 2000 (Invitrogen) according to the manufacturer's instructions. Each transfection contained $800 \mathrm{ng}$ of firefly luciferase reporter plasmid and $80 \mathrm{ng}$ of pRL-SV40 plasmid. Twenty-four hours after the transfection, the cells were treated with different concentrations of EGCG for $24 \mathrm{~h}$. Cell lysates were analyzed for firefly and Renilla luciferase activities according to the Dual Luciferase Reporter Assay kit (Promega) protocol. The firefly luciferase activity was normalized to the value of pRL-SV40 activity for transfection efficiency.

Soft agar colony assay. To examine the anchorage-independent growth, lung cancer cells were suspended $(10,000$ cells $/ \mathrm{ml})$ in $1 \mathrm{ml}$ of $0.3 \%$ agar with Eagle's basal medium containing $10 \%$ FBS, $1 \%$ antibiotics, and different concentrations of EGCG $(0,1,5,10,20$ and $40 \mu \mathrm{mol} / \mathrm{l})$ overlaid into 6-well plates containing a $0.6 \%$ agar base. The cultures were maintained in a $37^{\circ} \mathrm{C}, 5 \% \mathrm{CO}_{2}$ incubator for $1-2$ weeks, and then colonies were counted under a microscope using the Image-Pro Plus software program (Media Cybernetics, Silver Spring, MD, USA).

Ubiquitination assay. $\mathrm{H} 1299$ and $293 \mathrm{~T}$ cells were co-transfected with $2 \mu \mathrm{g}$ of GFP-p53, $1 \mu \mathrm{g}$ of HA-MDM2 and $0.5 \mu \mathrm{g}$ His-Ub plasmids. Twenty-four hours after the transfection, the cells were treated with different concentrations of EGCG for a further $24 \mathrm{~h}$. Subsequently, $20 \mu \mathrm{M}$ of MG132 was added to the culture medium for $6 \mathrm{~h}$ prior to harvesting. Cells were split into two aliquots, one for immunoblotting and the other for a ubiquitination assay. For the ubiquitination assay, we first used the anti-GFP antibody to immunoprecipitate p53 and then detected the ubiquitination using a His-tag antibody. A549 cells were pre-treated with EGCG for $24 \mathrm{~h}$ and MG132 for $6 \mathrm{~h}$. Endogenous p53 was immunoprecipitated from $1 \mathrm{mg}$ of protein lysate using the p53 antibody and then immunoblotted with the anti-ubiquitin antibody to capture polyubiquitinated $\mathrm{p} 53$.

Statistical analysis. All the statistical analyses were performed using the SPSS software (version 13.0). The experiments were performed in triplicate. The quantitative data are expressed as mean values \pm standard deviation. The significant differences between two groups were assessed by a two-tailed Student's t-test. $\mathrm{P}<0.05$ was considered to represent a statistically significant difference. 
A
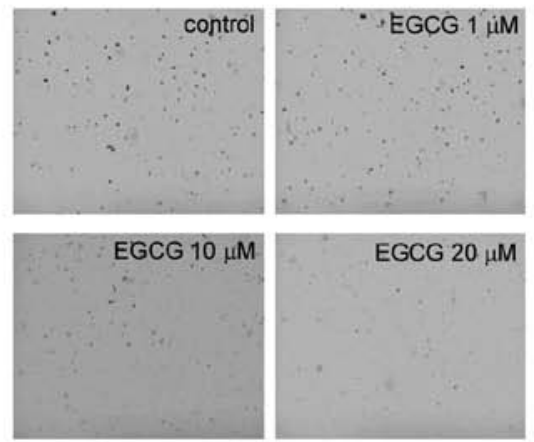

B
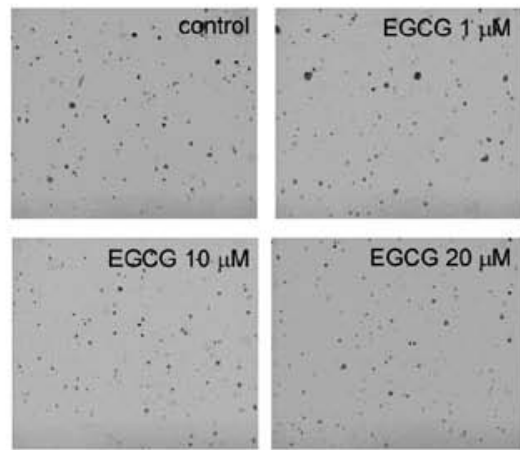

C
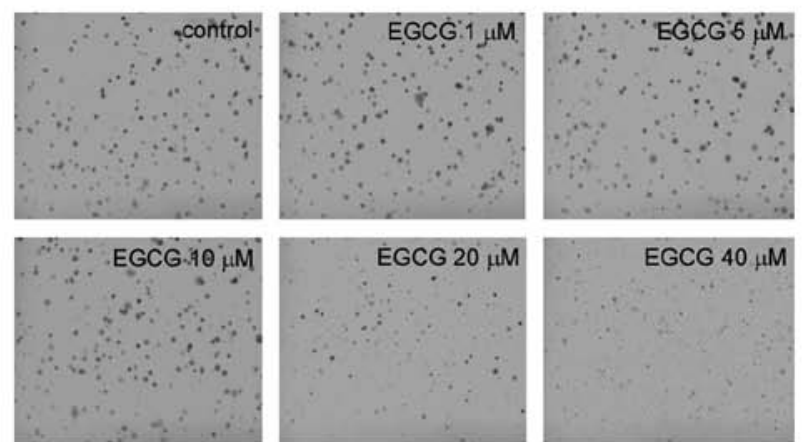
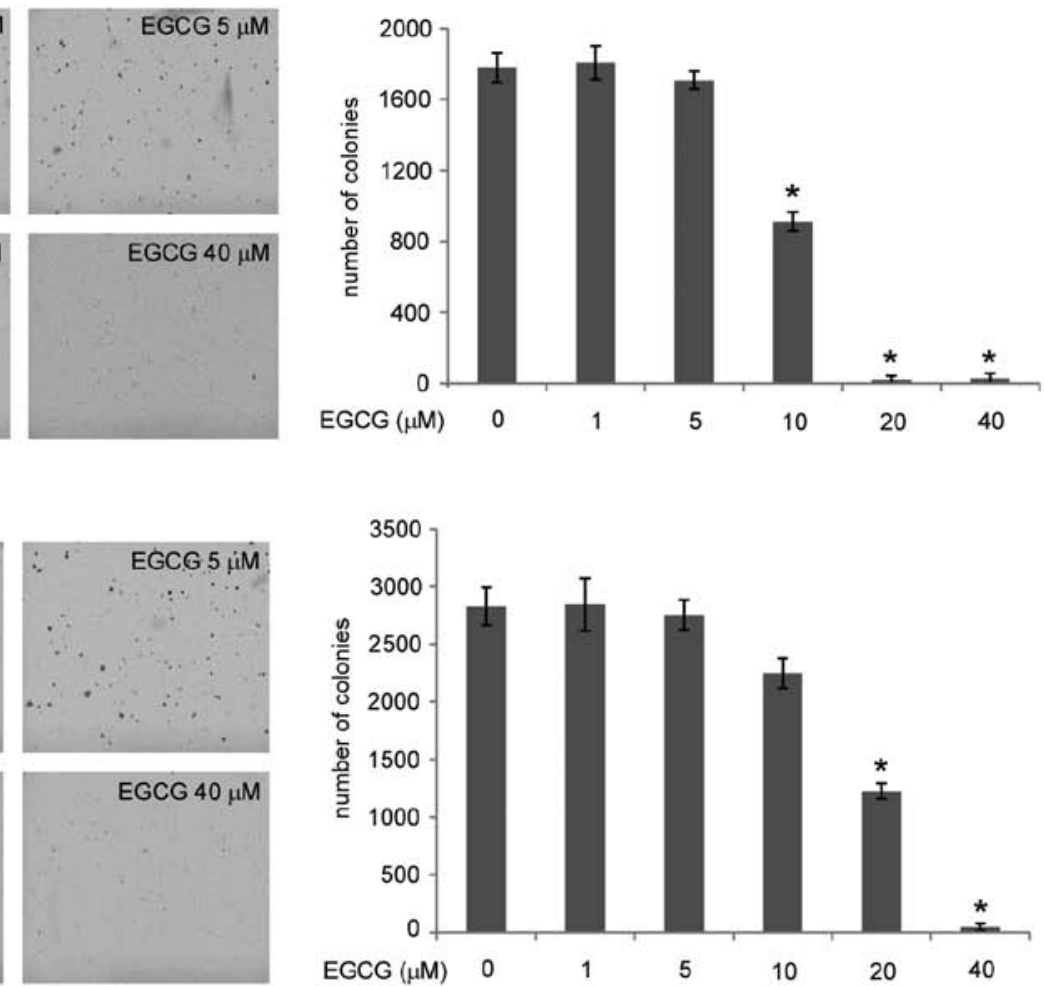

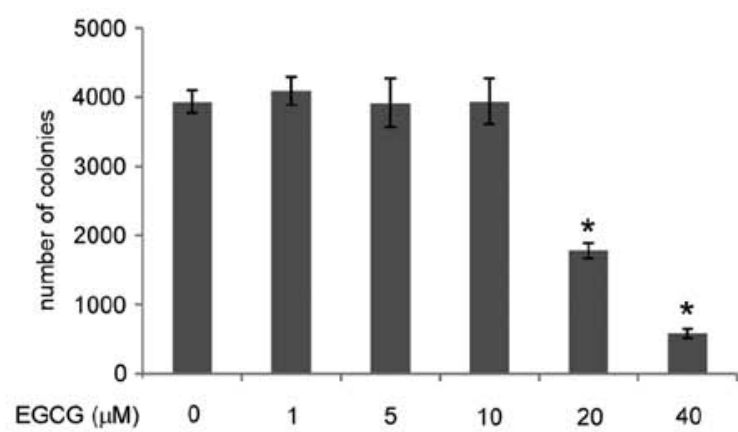

Figure 1. Inhibitory effects of EGCG on anchorage-independent growth of human lung cancer cells. EGCG inhibited anchorage-independent growth in a panel of human lung cancer cells, including (A) H1650, (B) A549 and (C) H460 cells. A colony formation assay was performed as described in Materials and methods. Data shown are the colony formation ability of human lung cancer cells treated with different concentrations of EGCG compared with the dimethyl sulfoxide-treated group. The average colony number was calculated from three separate experiments. Columns, means of the number of colonies as determined from three independent experiments; bars, standard deviation. "P<0.01 significant suppression of colony formation by EGCG.

\section{Results}

EGCG inhibits the anchorage-independent growth of human lung cancer cells. Initially, we investigated the effects of EGCG on the anchorage-independent growth of three different types of human lung cancer cell lines: A549, H1650 and H460. The results showed that these three cell lines have different sensitivity to EGCG. At low concentrations $(1-5 \mu \mathrm{M})$, EGCG does not substantially inhibit the anchorageindependent growth of these lung cancer cells. In H1650 cells, EGCG potently inhibits the anchorage-independent growth at the concentration of $10 \mu \mathrm{M}$, and almost no colonies were formed at $20 \mu \mathrm{M}$ (Fig. 1A). In A549 and H460 cells, EGCG showed clear inhibitory effects against anchorage-independent growth at the concentration of $20 \mu \mathrm{M}$ (Fig. 1B and C, respectively). These results indicate that EGCG inhibits the anchorage-independent growth of human lung cancer cells in a dose-dependent manner.

EGCG induces p53 accumulation and upregulates its target genes. Based on the results of the soft agar experiments, we detected the effects of EGCG on p53 expression in three p53-wild type human lung cancer cells: A549 (Fig. 2A), H1650 (Fig. 2B) and H460 (Fig. 2C). Our results showed that EGCG dose-dependently increased the endogenous p53 expression. At the concentration of $5 \mu \mathrm{M}$, the expression of p53 was substantially increased following the treatment of EGCG for $24 \mathrm{~h}$. In addition, we investigated the effects of EGCG on its downstream target genes, p21 and MDM2. Similar to the effect on p53, EGCG enhanced the expression levels of p21 
A

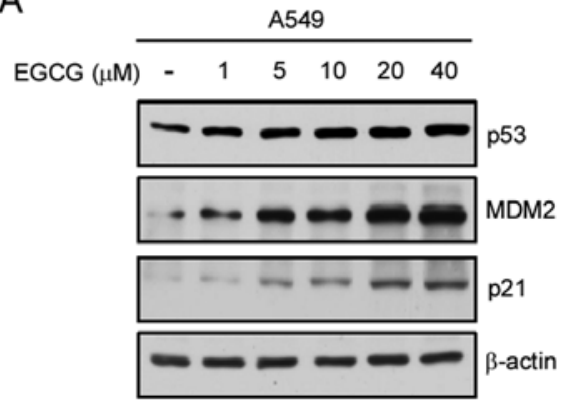

B

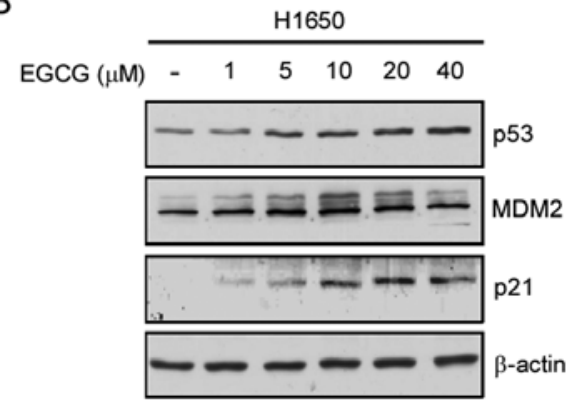

C

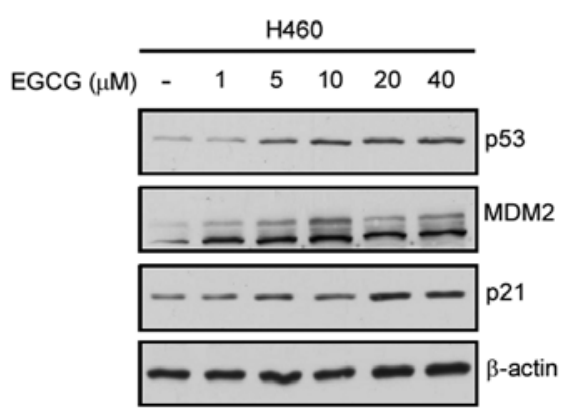

Figure 2. EGCG induces p53 accumulation and upregulates its target genes. EGCG upregulates endogenous p53, p21 and MDM2 expression in a dose-dependent manner in human lung cancer (A) H1650, (B) A549 and (C) H460 cells. The cells were seeded in 10-cm dishes and treated with varying concentrations of EGCG for $24 \mathrm{~h}$. The cells were then harvested, and protein levels were determined by western blot analysis.
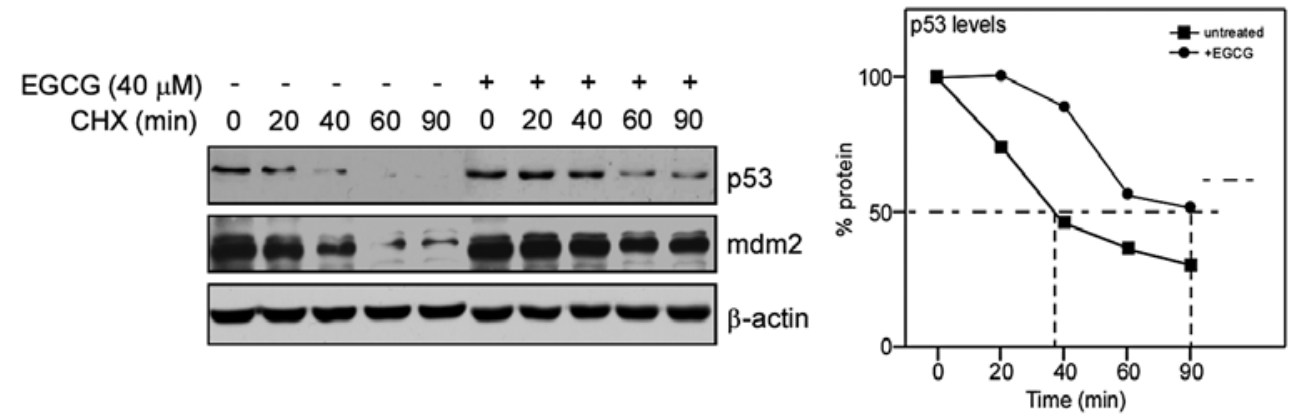

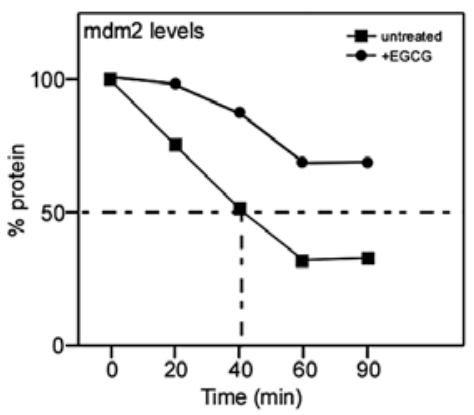

Figure 3. EGCG promotes p53 and MDM2 protein stability. A549 cells were seeded in 6-cm dishes, and following culture with $40 \mu \mathrm{M}$ of EGCG for $24 \mathrm{~h}$, $20 \mu \mathrm{g} / \mathrm{ml} \mathrm{CHX}$ was added to the medium. The cells were harvested at 0, 20, 40, 60 and $90 \mathrm{~min}$. p53 and MDM2 expression levels were determined by western blot analysis and were quantified by densitometry.

and MDM2. These data indicate that EGCG increases the expression of p53 and its target genes.

EGCG promotes the stability of p53 and MDM2. As a shortlived protein, $\mathrm{p} 53$ is degraded quickly by ubiquitination under normal physiologic status. Enhancement of protein stability is the first step for $\mathrm{p} 53$ to perform its functions. To expose the mechanism of $\mathrm{p} 53$ upregulation by EGCG, we first set up a halflife assay to investigate the effects of EGCG on p53 stability. We adopted CHX treatment to block the protein synthesis in A549 cells, and western blotting to detect the expression levels of p53 following treatment with EGCG for $24 \mathrm{~h}$. We found that EGCG significantly upregulated the half-life of p53 from $\sim 40 \mathrm{~min}$ to $>90 \mathrm{~min}$. Meanwhile, as its target gene and major negative regulatory factor, the half-life of MDM2 also clearly increased after EGCG treatment (Fig. 3). These results demonstrate that EGCG promotes the stability of p53 and MDM2.

EGCG promotes nuclear localization and activity of $p 53$. Ubiquitination of $\mathrm{p} 53$ results in targeting either for proteasomal degradation or nuclear export. As an important transcriptional factor, p53 stability and nuclear localization are essential for its tumor suppressor function (34), In the nucleus, as a result of MDM2-mediated ubiquitination, p53 is transported into the cytoplasm or is degraded by the $26 \mathrm{~S}$ proteasome. Both in the nucleus and in the cytoplasm, the phosphorylation of p53 is considered to be a counteractive post-translational modifica- tion that promotes 553 stability and transactivation. Previous studies have reported that the phosphorylation of p53 at Ser15 or Ser20 increases p53 stability by disrupting the interaction between 553 and MDM2 $(42,43)$. In the present study, we found that EGCG increased Ser15 and Ser20 phosphorylation of p53 in a dose-dependent manner (Fig. 4A). We also confirmed the localization of $\mathrm{p} 53$ and MDM2 by cellular fractionation. As shown in Fig. 4B, EGCG treatment only slightly promoted the accumulation of p53 in the cytoplasm. However, in the nucleus, EGCG dose-dependently increased the accumulation of p53, with a significant increase at the concentration of $20 \mu \mathrm{M}$. By contrast, MDM2 in the nucleus was mildly decreased as the concentration of EGCG increased. Furthermore, we tested whether EGCG treatment affects the transactivation ability of p53 using a Dual Luciferase Reporter Assay system. As shown in Fig. 4C, the transactivation ability of p53 was significantly elevated following EGCG treatment. At the concentration of $20 \mu \mathrm{M}$, the luciferase activity increased nearly 3 times. These results suggest that EGCG potentiates p53 function in human lung cancer cells.

EGCG inhibits proteasomal degradation-dependent $p 53$ ubiquitination. Based on the observation that EGCG promotes the stability of $\mathrm{p} 53$, we further investigated the effects of EGCG on p53 ubiquitination. Plasmids GFP-p53 $(3 \mu \mathrm{g})$, HA-MDM2 $(0.5 \mu \mathrm{g})$ and His-Ub $(0.5 \mu \mathrm{g})$ were co-transfected into $293 \mathrm{~T}$ cells, which were used as an overexpression system. 
A

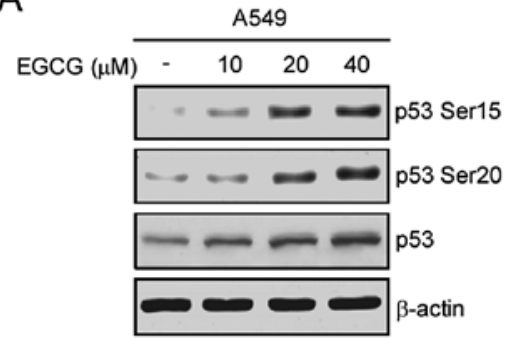

B

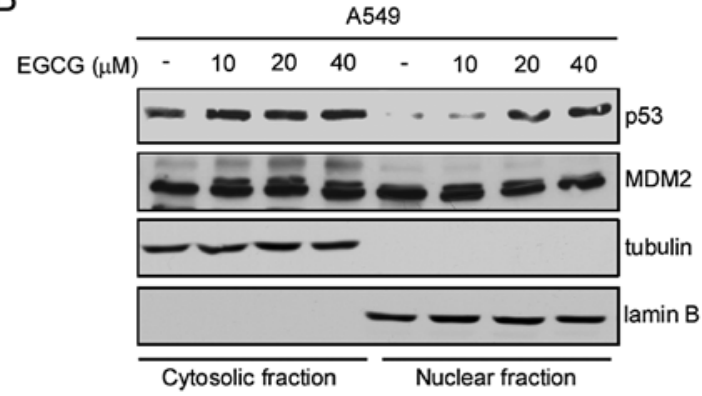

C

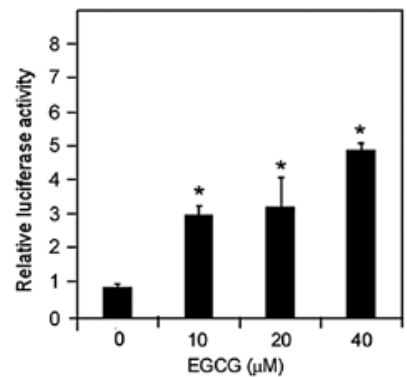

Figure 4. EGCG promotes nuclear localization and activity of p53. (A) EGCG increases p53 Ser15 and Ser20 phosphorylation. A549 cells were treated with EGCG for $24 \mathrm{~h}$ and the whole cell lysates were subjected to western blot analysis using the indicated antibodies. (B) p53 was upregulated and translocated to the nucleus upon EGCG treatment. Endogenous 553 and MDM2 in both cytoplasm (non-NE) and nucleus (NE) of A549 cells, which were treated with EGCG for $24 \mathrm{~h}$, were detected using anti-p53, MDM2, tubulin and lamin B antibodies for western blotting. (C) EGCG upregulated p53 transcriptional activity. A549 cells were transfected with the indicated plasmids. EGCG treatment and luciferase reporter assays were performed as described. The relative luciferase activity was normalized to the value of Renilla activity.

A

\begin{tabular}{lcccc} 
& \multicolumn{4}{c}{ IP: GFP } \\
\cline { 2 - 5 } GFP-p53 & + & + & + & + \\
HA-MDM2 & + & + & + & + \\
His-Ub & + & + & + & + \\
EGCG $(\mu M)$ & - & 10 & 20 & 40
\end{tabular}
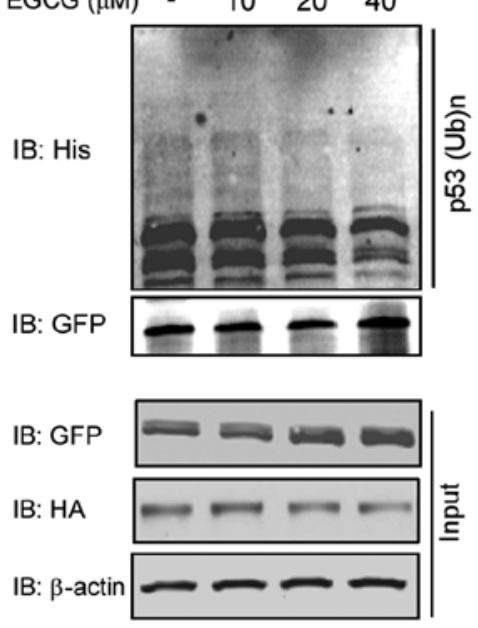

B

\begin{tabular}{lcccc} 
& \multicolumn{4}{c}{ IP: GFP } \\
\cline { 2 - 5 } GFP-p53 & + & + & + & + \\
HA-MDM2 & + & + & + & + \\
His-Ub & + & + & + & + \\
EGCG $(\mu M)$ & - & 10 & 20 & 40
\end{tabular}

IB: His

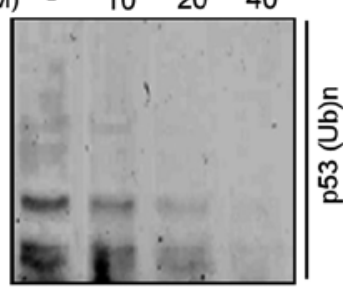

IB: GFP

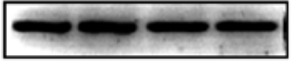

IB: GFP

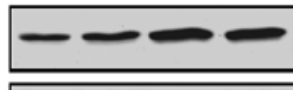

IB: HA

IB: $\beta$-actin
C

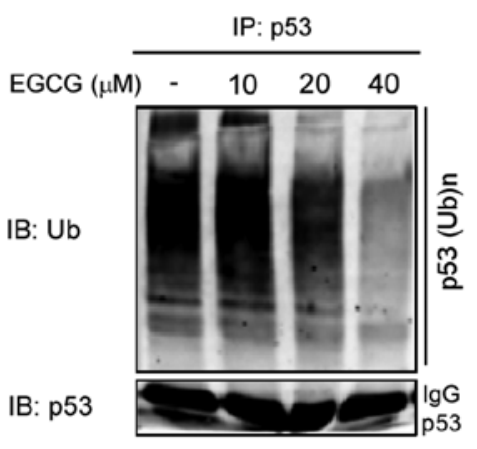

IB: p53

IB: $\beta$-actin

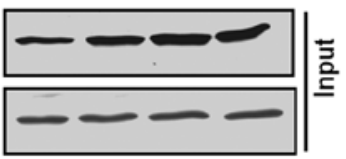

Figure 5. EGCG inhibits p53 ubiquitination. (A) 293T and (B) H1299 cells were co-transfected with different expression vectors and treated with EGCG as described. Cell lysates were immunoprecipitated with GFP antibody and analyzed by immunoblotting with anti-His-tag antibody to capture polyubiquitinated p53. The same cell lysates were subjected to western blotting to determine the expression levels of p53 and MDM2 protein. (C) EGCG inhibited endogenous p53 ubiquitination. A549 cells were treated with or without EGCG as indicated. p53 was immunoprecipitated from 1 mg of cell lysate and immunoblotted with anti-ubiquitin antibody to detect ubiquitinated p53.

As shown in Fig. 5A, EGCG treatment substantially decreased the ubiquitination of $\mathrm{p} 53$ with significant inhibition observed at the concentration of $20 \mu \mathrm{M}$. We also tested this phenomenon by transfection of these plasmids in p53-deficient H1299 cells. From the results shown in Fig. 5B, EGCG also dose-dependently inhibited the ubiquitination of $\mathrm{p} 53$. In addition to the overexpression system, we also studied the effects of EGCG on the regulation of endogenous p53 ubiquitination (Fig. 5C). In the p53-wild type A549 cell line treated with EGCG at the concentration of $20 \mu \mathrm{M}$ for $24 \mathrm{~h}$, the ubiquitination of $\mathrm{p} 53$ was markedly decreased. These results suggest that EGCG inhibits p53 ubiquitination.
EGCG inhibits the interaction of $p 53$ and MDM2. The results of the above experiments demonstrated that EGCG inhibits the ubiquitination of $\mathrm{p} 53$, promotes its stability and increases its expression. As a downstream target gene of p53, the expression levels of MDM2 also increased with the accumulation of p53 in the nucleus. However, as an important negative regulator of $\mathrm{p} 53$, the increase in MDM2 expression may cause the ubiquitination and subsequent degradation of $\mathrm{p} 53$. The previous results confirmed that EGCG markedly inhibits the ubiquitination of p53 even in the case of MDM2 overexpression. Therefore, studies were performed to further investigate the effects of EGCG on the interaction between MDM2 and 
A

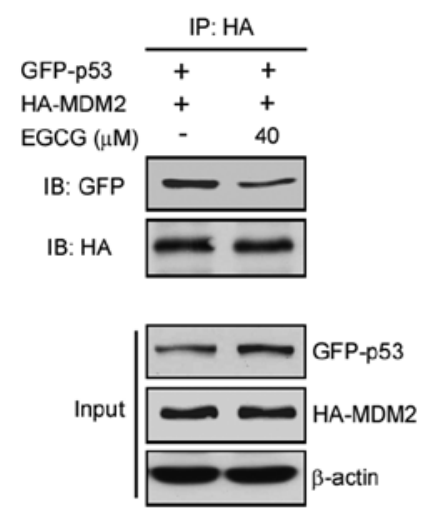

B
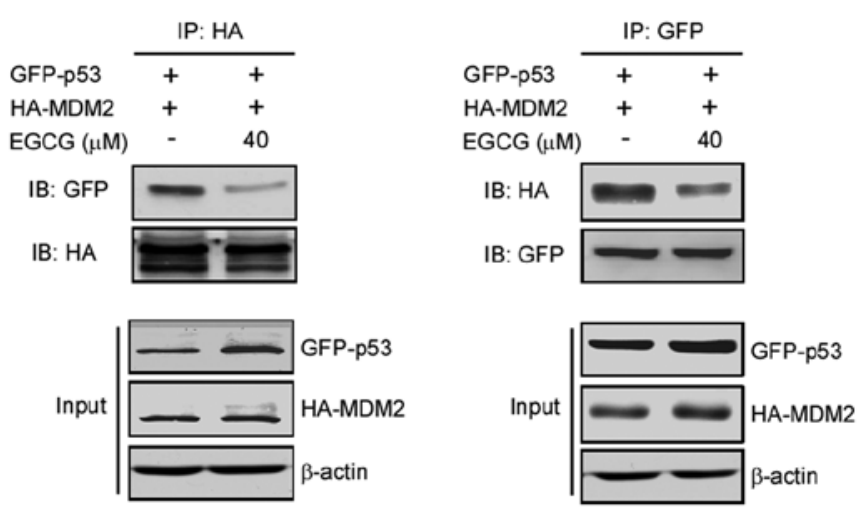

C
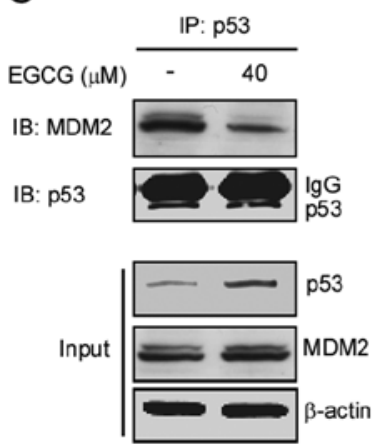

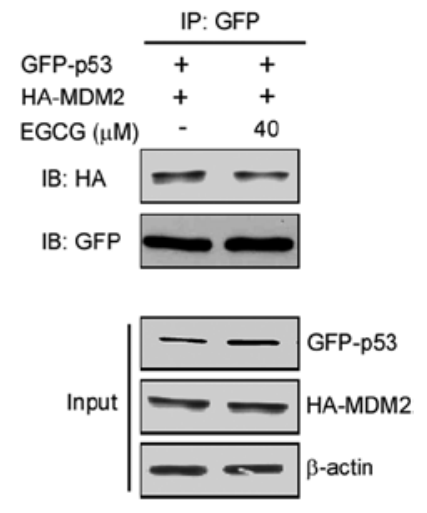

observed similar results in which EGCG treatment inhibited MDM2 binding with p53 (Fig. 6C). These results indicate that EGCG suppresses MDM2-mediated p53 ubiquitination by disrupting their interaction.

\section{Discussion}

The natural compound EGCG is the major polyphenol component of green tea. It has been extensively studied due to its relatively high abundance and strong epidemiologic evidence of cancer-preventive activity $(2,3)$. Although several reports have shown that EGCG exerts its anticancer activity by targeting specific cell signaling pathways, the underlying mechanism is not yet fully understood.

Previous reports revealed that EGCG treatment induces lung cancer cell apoptosis or cell cycle arrest $(18,35)$. Other groups also found that the tumor suppressor gene p53 may be involved in the antitumor activity of EGCG since cancer cells expressing wild-type p53 are more sensitive to EGCG treatment compared with p53-null or p53 knock down cancer cells $(36,40)$. In the present study, we first examined the antitumor effects of EGCG in several human lung cancer cells that express wild-type p53 by soft agar assay. Our results demonstrated that EGCG inhibits the anchorage-independent growth of human lung cancer cells in a dose-dependent manner. Western blotting data indicated that EGCG promotes the accumulation of wild-type p53 and its target genes, p21 and MDM2. Our data demonstrated and corroborated other studies showing that the effect of EGCG against lung cancer may partly depend on p53 activity.

To further investigate $\mathrm{p} 53$ stability mediated by EGCG, the effects of EGCG on the half-life of p53 and MDM2 were examined using CHX treatment. We found that EGCG significantly upregulated the half-life of p53 from approximately $40 \mathrm{~min}$ to $90 \mathrm{~min}$, as well as that of its target gene, MDM2, from approximately $40 \mathrm{~min}$ to over $90 \mathrm{~min}$. These results indicate that p53 is a potential anticancer target mediated by EGCG.

The activity and localization of p53 are mainly regulated by post-translational modifications, such as phosphorylation, acetylation and ubiquitination $(27,28,34)$. Accumulating evidence demonstrates that p53 phosphorylation at Ser15 and Ser20 attenuates the binding of p53 to MDM2 and disrupts MDM2-mediated p53 ubiquitination both in vivo and in vitro $(42,43)$. In our study, we demonstrated that the increased p53 expression was accompanied by the phosphorylation of p53 at Ser15 and Ser20. In addition, by extracting the cytosolic and nuclear fractions, we further determined p53 subcellular localization upon EGCG treatment. The results clearly showed that EGCG potently induced nuclear accumulation of p53 in a dose-dependent manner while, at the same time, the expression levels of MDM2 in the nucleus appeared slightly decreased. Therefore, we hypothesized that EGCGinduced stabilization and nuclear accumulation of p53 might induce an increase in its transcriptional activity and result in an upregulation of its target genes, p21 and MDM2. Thus, we tested p 53 transcriptional activity by setting up a reporter gene assay. Our data confirmed the hypothesis that EGCG treatment increases p 53 transcriptional activity.

\section{MDM2, a downstream target gene of $\mathrm{p} 53$, is the major
E3 ligase responsible for regulating p53 polyubiquitination \\ MDM2, a downstream target gene of p53, is the major
E3 ligase responsible for regulating p53 polyubiquitination}

p53. The plasmids GFP-p53 ( $2 \mu \mathrm{g})$ and HA-MDM2 $(2 \mu \mathrm{g})$ were co-transfected into 293T and H1299 cells. Following treatment with $40 \mu \mathrm{M}$ EGCG for $24 \mathrm{~h}$, co-immunoprecipitation was performed to detect the interaction between MDM2 and p53. As shown in Fig. 6A and B, in the overexpression systems 293T and H1299, EGCG treatment resulted in substantial inhibition of the interaction between p53 and MDM2. In A549, we 
and targeting p53 for proteasomal degradation. Therefore, enhanced p53 activity causes increased expression of its own negative regulator, MDM2, thereby forming an autoregulatory feedback loop. In the present study, we found that EGCG treatment sustainably increased p53 expression, accompanied by MDM2 upregulation. To further investigate the underlying mechanism, we detected p53 polyubiquitination by immunoprecipitation. Although EGCG treatment increased MDM2 protein levels, MDM2-mediated p53 ubiquitination was markedly decreased. Furthermore, our co-immunoprecipitation data showed that EGCG treatment disrupts the interaction between p53 and MDM2. This observation suggests that upon EGCG treatment, MDM2 acts only as a target gene and is upregulated by $\mathrm{p} 53$, and that its activity to induce p53 degradation is suppressed.

Collectively, our study identifies p53 as a potential target of EGCG to execute its cancer-preventive activity, and that the natural compound EGCG, inhibits the anchorage-independent growth of human lung cancer cells by promoting p53 stability/activity and by inhibiting MDM2-mediated p53 ubiquitination and degradation. Our study provides a new mechanism to explain the chemopreventive effect of EGCG on cancer; further research is required to provide evidence for the clinical use of EGCG in cancer prevention and therapy.

\section{Acknowledgements}

This study was supported by the Nature Scientific Foundation of Hunan Province (grant no. 08JJ6010) and the Research Program from the Science Technology Department of Hunan Province (grant no. 2012FJ4076).

\section{References}

1. Chen L and Zhang HY: Cancer preventive mechanisms of the green tea polyphenol (-)-epigallocatechin-3-gallate. Molecules 12: 946-957, 2007.

2. Johnson R, Bryant S and Huntley AL: Green tea and green tea catechin extracts: an overview of the clinical evidence. Maturitas 73: 280-287, 2012.

3. Fujiki $\mathrm{H}$ and Suganuma M: Green tea: an effective synergist with anticancer drugs for tertiary cancer prevention. Cancer Lett 324: $119-125,2012$.

4. Fujiki H, Imai K, Nakachi K, Shimizu M, Moriwaki H and Suganuma M: Challenging the effectiveness of green tea in primary and tertiary cancer prevention. J Cancer Res Clin Oncol 138: 1259-1270, 2012

5. Surh YJ: Cancer chemoprevention with dietary phytochemicals. Nat Rev Cancer 3: 768-780, 2003.

6. Yang CS and Wang X: Green tea and cancer prevention. Nutr Cancer 62: 931-937, 2010.

7. Singh BN, Shankar S and Srivastava RK: Green tea catechin, epigallocatechin-3-gallate (EGCG): mechanisms, perspectives and clinical applications. Biochem Pharmacol 82: 1807-1821, 2011.

8. Khan N, Afaq F, Saleem M, Ahmad N and Mukhtar H: Targeting multiple signaling pathways by green tea polyphenol (-)-epigallocatechin-3-gallate. Cancer Res 66: 2500-2505, 2006.

9. Shimizu M, Deguchi A, Lim JT, Moriwaki H, Kopelovich L and Weinstein IB: (-)-Epigallocatechin gallate and polyphenon E inhibit growth and activation of the epidermal growth factor receptor and human epidermal growth factor receptor-2 signaling pathways in human colon cancer cells. Clin Cancer Res 11 : 2735-2746, 2005.

10. Yang CS, Wang H, Li GX, Yang Z, Guan F and Jin H: Cancer prevention by tea: evidence from laboratory studies. Pharmacol Res 64: 113-122, 2011.
11. Levites Y, Amit T, Youdim MB and Mandel S: Involvement of protein kinase $\mathrm{C}$ activation and cell survival/cell cycle genes in green tea polyphenol (-)-epigallocatechin 3-gallate neuroprotective action. J Biol Chem 277: 30574-30580, 2002.

12. Relat J, Blancafort A, Oliveras G, Cufi S, Haro D, Marrero PF and Puig T: Different fatty acid metabolism effects of (-)-epigallocatechin-3-gallate and C75 in adenocarcinoma lung cancer. BMC Cancer 12: 280, 2012

13. Sah JF, Balasubramanian S, Eckert RL and Rorke EA: Epigallocatechin-3-gallate inhibits epidermal growth factor receptor signaling pathway. Evidence for direct inhibition of ERK1/2 and AKT kinases. J Biol Chem 279: 12755-12762, 2004.

14. Kanwar J, Taskeen M, Mohammad I, Huo C, Chan TH and Dou QP: Recent advances on tea polyphenols. Front Biosci (Elite Ed) 4: 111-131, 2012.

15. Pianetti S, Guo S, Kavanagh KT and Sonenshein GE: Green tea polyphenol epigallocatechin-3 gallate inhibits Her-2/neu signaling, proliferation, and transformed phenotype of breast cancer cells. Cancer Res 62: 652-655, 2002.

16. Yang F, Oz HS, Barve S, de Villiers WJ, McClain CJ and Varilek GW: The green tea polyphenol (-)-epigallocatechin3-gallate blocks nuclear factor-kappa B activation by inhibiting I kappa B kinase activity in the intestinal epithelial cell line IEC-6. Mol Pharmacol 60: 528-533, 2001.

17. Ahmad N, Feyes DK, Nieminen AL, Agarwal R and Mukhtar H: Green tea constituent epigallocatechin-3-gallate and induction of apoptosis and cell cycle arrest in human carcinoma cells. J Natl Cancer Inst 89: 1881-1886, 1997.

18. Saha A, Kuzuhara T, Echigo N, Suganuma M and Fujiki H: New role of (-)-epicatechin in enhancing the induction of growth inhibition and apoptosis in human lung cancer cells by curcumin. Cancer Prev Res 3: 953-962, 2010.

19. Chang CM, Chang PY, Tu MG, et al: Epigallocatechin gallate sensitizes CAL-27 human oral squamous cell carcinoma cells to the anti-metastatic effects of gefitinib (Iressa) via synergistic suppression of epidermal growth factor receptor and matrix metalloproteinase-2. Oncol Rep 28: 1799-1807, 2012.

20. Deng YT and Lin JK: EGCG inhibits the invasion of highly invasive CL1-5 lung cancer cells through suppressing MMP-2 expression via JNK signaling and induces G2/M arrest. J Agric Food Chem 59: 13318-13327, 2011.

21. Jung YD and Ellis LM: Inhibition of tumour invasion and angiogenesis by epigallocatechin gallate (EGCG), a major component of green tea. Int J Exp Pathol 82: 309-316, 2001.

22. Liu LC, Tsao TC, Hsu SR, Wang HC, Tsai TC, Kao J and Way TD: EGCG inhibits transforming growth factor- $\beta$-mediated epithelial-to-mesenchymal transition via the inhibition of Smad2 and Erk1/2 signaling pathways in nonsmall cell lung cancer cells. J Agric Food Chem 60: 9863-9873, 2012.

23. Singh T and Katiyar SK: Green tea catechins reduce invasive potential of human melanoma cells by targeting COX-2, PGE2 receptors and epithelial-to-mesenchymal transition. PLoS One 6: e25224, 2011.

24. Watanabe T, Kuramochi $\mathrm{H}$, Takahashi A, et al: Higher cell stiffness indicating lower metastatic potential in B16 melanoma cell variants and in (-)-epigallocatechin gallate-treated cells. J Cancer Res Clin Oncol: Feb 2, 2012 (Epub ahead of print).

25. Charvet C, Wissler M, Brauns-Schubert P, et al: Phosphorylation of Tip60 by GSK-3 determines the induction of PUMA and apoptosis by p53. Mol Cell 42: 584-596, 2011.

26. Dai C, Tang Y, Jung SY, Qin J, Aaronson SA and Gu W: Differential effects on p53-mediated cell cycle arrest vs. apoptosis by p90. Proc Natl Acad Sci USA 108: 18937-18942, 2011.

27. Dai $\mathrm{C}$ and $\mathrm{Gu} \mathrm{W}$ : $\mathrm{p} 53$ post-translational modification: deregulated in tumorigenesis. Trends Mol Med 16: 528-536, 2010.

28. Kruse JP and Gu W: SnapShot: p53 post-translational modifications. Cell 133: 930-930.e1, 2008.

29. Kruse JP and Gu W: Modes of p53 regulation. Cell 137: 609-622, 2009.

30. Munoz-Fontela C, Gonzalez D, Marcos-Villar L, et al: Acetylation is indispensable for p53 antiviral activity. Cell Cycle 10: 3701-3705, 2011.

31. Sakaguchi K, Herrera JE, Saito S, et al: DNA damage activates p53 through a phosphorylation-acetylation cascade. Genes Dev 12: 2831-2841, 1998.

32. Jimenez GS, Khan SH, Stommel JM and Wahl GM: p53 regulation by post-translational modification and nuclear retention in response to diverse stresses. Oncogene 18: 7656$7665,1999$. 
33. Lee $\mathrm{JT}$ and $\mathrm{Gu} \mathrm{W}$ : The multiple levels of regulation by $\mathrm{p} 53 \mathrm{ubiq}-$ uitination. Cell Death Differ 17: 86-92, 2010.

34. Yuan J, Luo K, Zhang L, Cheville JC and Lou Z: USP10 regulates p53 localization and stability by deubiquitinating p53. Cell 140: 384-396, 2010.

35. Amin AR, Wang D, Zhang H, et al: Enhanced anti-tumor activity by the combination of the natural compounds (-)-epigallocatechin-3-gallate and luteolin: potential role of p53. J Biol Chem 285: 34557-34565, 2010.

36. Hastak K, Gupta S, Ahmad N, Agarwal MK, Agarwal ML and Mukhtar H: Role of p53 and NF-kappaB in epigallocatechin3-gallate-induced apoptosis of LNCaP cells. Oncogene 22: 4851-4859, 2003.

37. Lee MH, Han DW, Hyon SH and Park JC: Apoptosis of human fibrosarcoma HT-1080 cells by epigallocatechin-3-O-gallate via induction of p53 and caspases as well as suppression of $\mathrm{Bcl}-2$ and phosphorylated nuclear factor-kappaB. Apoptosis 16 : 75-85, 2011.

38. Qin J, Chen HG, Yan Q, et al: Protein phosphatase-2A is a target of epigallocatechin-3-gallate and modulates p53-Bak apoptotic pathway. Cancer Res 68: 4150-4162, 2008.
39. Hastak K, Agarwal MK, Mukhtar H and Agarwal ML: Ablation of either p21 or Bax prevents p53-dependent apoptosis induced by green tea polyphenol epigallocatechin-3-gallate. FASEB J 19: 789-791, 2005

40. Thakur VS, Ruhul Amin AR, Paul RK, et al: p53-Dependent p21-mediated growth arrest pre-empts and protects HCT116 cells from PUMA-mediated apoptosis induced by EGCG. Cancer Lett 296: 225-232, 2010.

41. Zhao BX, Chen HZ, Lei NZ, et al: p53 mediates the negative regulation of MDM2 by orphan receptor TR3. EMBO J 25: 5703-5715, 2006.

42. Chehab NH, Malikzay A, Stavridi ES and Halazonetis TD: Phosphorylation of Ser-20 mediates stabilization of human p53 in response to DNA damage. Proc Natl Acad Sci USA 96: 13777-13782, 1999.

43. Unger T, Juven-Gershon T, Moallem E, et al: Critical role for Ser20 of human $\mathrm{p} 53$ in the negative regulation of $\mathrm{p} 53$ by Mdm2. EMBO J 18: 1805-1814, 1999. 